\title{
A linked stress release model for historical Japanese earthquakes: coupling among major seismic regions
}

\author{
Chunsheng $\mathrm{Lu}^{1}$, David Harte ${ }^{1}$, and Mark Bebbington ${ }^{2}$ \\ ${ }^{1}$ MCS, Victoria University of Wellington, PO Box 600, Wellington, New Zealand \\ ${ }^{2}$ IIS\&T, Massey University, Private Bag 11222, Palmerston North, New Zealand \\ (Received October 28, 1998; Revised August 6, 1999; Accepted August 9, 1999)
}

\begin{abstract}
A linked stress release model is proposed for the analysis of spatial interaction of earthquake occurrences through stress transfer within a large area of the Earth's crust. As an example, the model is used for statistical analysis for the Japanese historical earthquakes in central Japan and offshore in the Nankai and Sagami troughs with magnitude $M \geq 6.5$ during the period from 1400 to 1997 . This area is divided into four smaller regions of roughly comparable size and activity. Based on the Akaike information criterion (AIC), the results demonstrate the existence of coupling between certain of the regions. With the evidence that the crust may lie in a near-critical state, this has implications for the possible triggering of earthquakes at long distances from the origin event. In particular, we find evidence for the dependence of Nankai trough events on the Chubu/Kinki triangle region, whose events are themselves dependent on the the Fossa Magna/Sagami trough. Evidence for the validity of the model includes simulation results indicating that the model had a higher forecast hazard post-1991 for an event in the Chubu/Kinki triangle region than did models not incorporating regional coupling.
\end{abstract}

\section{Introduction}

The elastic rebound theory proposed by Reid (1910) is one of a few classical models for earthquake mechanisms. This model indicates that elastic stress in a seismically active region accumulates due to movement of tectonic plates, and is released when the stress exceeds the strength of the medium. By fixing only strength or residual stress in the model, we obtain the modifications known as time- and slip-predictable models. Although these models have been widely used in long-term prediction, real sequences of large earthquakes are fundamentally more complicated. In particular, the elastic rebound theory suggests that a large earthquake should be followed by a period of quiescence, whereas in reality a strong earthquake can be followed by a period of activation and sometimes another earthquake of comparable magnitude (Gabrielov and Newman, 1994). Although the stress or strain field within a region can be extracted from various kinds of information, the temporal variations of seismic activity may most directly reflect the nature of earthquakegenerating stress (Zhao et al., 1990).

Through a development of the stochastic (Markov) model for the occurrence of main-sequence earthquakes suggested by Knopoff (1971), Vere-Jones (1978) proposed the stress release model, a stochastic version of the elastic rebound theory, incorporating a deterministic build-up of stress within a region and its stochastic release through earthquakes. The stress release model has been applied to the statistical analysis of several historical earthquake catalogs from China, Persia and Japan (Vere-Jones and Deng, 1988; Zheng and

Copy right $(\mathrm{C})$ The Society of Geomagnetism and Earth, Planetary and Space Sciences (SGEPSS); The Seismological Society of Japan; The Volcanological Society of Japan; The Geodetic Society of Japan; The Japanese Society for Planetary Sciences.
Vere-Jones, 1991, 1994). In particular, it has been used to identify, with the aid of geological information, statistically distinct regions, in the sense that they are best fitted by different stress-release models.

One of the most interesting phenomena observed in the above process is that large events are noticeably often followed by large events quite distant from the first. This seems most plausibly consistent with a view of the events taking place in the Earth's crust as forming part of a tightly linked, near-critical process, exhibiting the self-similarity, long-range correlation and power-law distributions which are characteristic of a physical process in near-critical conditions (Vere-Jones, 1976; Takayasu, 1990; Kagan, 1994). Many recent studies focusing on non-linear aspects of earthquake dynamics have shown that the Earth's crust may lie in a critically stable state and that earthquakes are consistent with typical self-organized criticality phenomena (see, for example, Bak and Tang, 1989; Ito and Matsuzaki, 1990; Main, 1996). A recent modification to this view has been the introduction of the accelerated moment release model (Bufe and Varnes, 1993), in which the crust approaches criticality, resulting in a major event, and the cycle restarting. When observation is limited to major events, the result is strikingly similar to the stress release model. In systems of this sort, one obtains an increase in correlation length as criticality is approached (Sornette and Sammis, 1995), which argues for the existence of correlation in occurrences of major events over large regions.

It is now understood that there exists a class of models exhibiting self-organized criticality due to competition between local strengthening and weakening through interactions. For example, see the crack propagation model of earthquakes 
proposed by Chen et al. (1991). Particularly, in order to obtain a satisfactory power law for the magnitude-frequency distribution of earthquakes (Gutenberg and Richter, 1954), long-range interaction of elastic stress should be introduced in earthquake triggering models (see Hill et al., 1993). Shimazaki (1976b) calculated that intraplate events result from a perturbation of the stress level due to interplate events, while the regional tectonic stress is close to crustal strength before interplate events. Kanamori (1972b) observed that tectonic stress changes over relatively large regions following great interplate events. Pollitz and Sacks (1997) note that "The process of adding an additional load to a fault zone due to a nearby earthquake perturbs an ongoing reloading cycle driven by regional stress, moving up or delaying the inevitable rupture". Regional stress itself evolves over centuries, being the result of the cumulative effects of all previous earthquakes and, in long enough time frames, of changes in tectonic loading. On the other hand, the spatio-temporal complexity of seismicity is also closely related to fault zone heterogeneities (Ben-Zion and Rice, 1997). In this paper, in order to construct a model consistent with the above tenets, we will extend the stress release model to interactions among regions, and apply this new version to the statistical analysis of the latest Japanese historical earthquake catalog for central Japan and the Nankai and Sagami troughs.

It has been observed (Shimazaki, 1976b) that the spatial variation of intraplate seismicity is correlated with great interplate events. Shimazaki also questioned whether Sagami trough events as well as Nankai trough events might be involved. Kanaori et al. (1993) qualified this correlation further, describing a migration of seismicity along the Median Tectonic Line (MTL) from east to west, followed by an event in the Nankai trough. A microplate model with the object of providing a tectonic framework for coupling among the MTL, adjacent fault systems, and the Nankai trough was introduced by Kanaori et al. (1994). The object of this paper is similar, although the methodology is statistical in nature, with the specific objective of quantifying the coupling and assessing its significance.

\section{The Model}

In the univariate stress release model the key variable, or state, is the stress level in a region, which controls the probability of an earthquake occurring (Vere-Jones and Deng, 1988). This stress level $X(t)$ increases deterministically between earthquakes and is reduced stochastically as a result of earthquakes. The current value $X(t)$ can be represented in the form

$$
X(t)=X(0)+\rho t-S(t),
$$

where $X(0)$ is the initial value, $\rho$ is a constant loading rate from external tectonic forces, and $S(t)$ is the accumulated stress release from earthquakes within the region over the period $(0, t)$, that is, $S(t)=\sum_{t_{i}<t} S_{i}$, where $t_{i}$ and $S_{i}$ are the origin time and the stress release associated with the $i$ th earthquake.

The probability intensity of an earthquake occurrence is controlled by a hazard function $\Psi(x)$, with the interpretation that the probability of an event occurring in the time interval $(t, t+\Delta)$ is approximately $\Psi(X(t)) \Delta$ for small $\Delta$. There are a wide range of possibilities for the function $\Psi$. Obviously, it must be nondecreasing. A constant independent of $x$ would result in a random (Poisson) model of occurrences. Using

$$
\Psi(x)= \begin{cases}0 & x \leq x_{\mathrm{c}} \\ \infty & x>x_{\mathrm{c}}\end{cases}
$$

produces the time-predictable model (Shimazaki and Nakata, 1980), supposing a fixed crustal strength $x_{\mathrm{c}}$. An effective compromise (Zheng and Vere-Jones, 1991, 1994) between these extremes of behavior is the form $\Psi(x)=\exp (\mu+v x)$. It also represents the behavior that might be expected from a region with a locally heterogeneous strength. One might also consider that local stress is inhomogeneous (Shimazaki, 1976b). The stochastic nature of the process with an exponential hazard function is compatible with these sources of "noise". We can interpret the constant $\mu$ (or rather the parameter $\alpha$ that replaces it, see below) as effectively a parameter to be fitted for the unknown initial value of stress, while the constant $v$ is an amalgam of the strength and heterogeneity of the crust in the region. This can also be understood as a form of sensitivity to risk.

On the other hand, the distribution of the stress released in an event is assumed to be independent of the stress level itself. Although there may be a weak dependence, the resulting improvement in fit does not justify its inclusion in the model (Zheng and Vere-Jones, 1991). A more important factor in the Japanese context seems to be differences in the magnitude distribution by region, particularly between intraplate and interplate events (Kanaori et al., 1991, 1993, 1994). Since the evidence for a Gutenberg-Richter relation for $M \geq 6.4$ is unclear (Shimazaki, 1976a), we shall use the historical empirical distribution for each region when simulating the model forward for forecasting purposes.

The key enabling statistical analysis is that the data in historical earthquake catalogs can be treated as a point process in time-stress space with conditional intensity function

$$
\lambda(t)=\Psi(X(t))=\exp [\mu+v(X(0)+\rho t-S(t))] .
$$

Obviously, $X(0)$ can be absorbed into the other parameters, and so we obtain the form $\lambda(t)=\exp [\alpha+v(\rho t-S(t))]$, where $\alpha=\mu+v X(0)$. Estimates of the parameters can then be found by maximizing the log-likelihood function (see, for example, Daley and Vere-Jones, 1988). For comparison, we shall also consider two special cases: $\lambda(t)=\exp [\alpha]$ and $\lambda(t)=\exp [\alpha+\beta t]$. The first of these is the Poisson process (PP), and the second we shall call the Poisson process with trend (PWT).

Note that aftershocks are not considered in the model as the stress, and hence the hazard, is assumed to decrease after an event. Hence the model attempts only to represent main sequence events, and aftershocks must be carefully identified and removed from the data before numerical fitting can begin. Obviously, stress transfer and interaction can not be considered in the simple stress release model, and the earlier analyses concentrated on dealing with regional differences.

According to simulation results obtained from certain mechanical models, the elastic stress in a system as a whole will be changed, and a long-range redistribution of stress occurs, after an event (fracture or slip) (Lu et al., 1998; Shi 
et al., 1998). A similar phenomenon is encountered in the analysis of real earthquake catalogs, where a region has interactions with neighboring regions. In particular, Zheng and Vere-Jones (1994) noted some hints of clustering which relate particularly to some form of action at a distance, i.e., stress transfer and interaction. This motivates a modification of the stress release model as follows. The evolution of stress $X_{i}(t)$ in the $i$ th region can be rewritten as

$$
X_{i}(t)=X_{i}(0)+\rho_{i} t-\sum_{j} \theta_{i j} S^{(j)}(t),
$$

where $S^{(j)}(t)$ is the accumulated stress release in region $j$ over the period $(0, t)$, and the coefficient $\theta_{i j}$ measures the fixed proportion of stress drop, initiated in region $j$, which is transferred to region $i$. Here, $\theta_{i j}$ may be positive or negative, resulting in damping or excitation respectively. It is convenient, in ignoring aftershocks, to set $\theta_{i i}=1$ for all $i$. The new version we shall call a linked (or coupled) stress release model. If $\theta_{i j}=0$ for all $i \neq j$, the model is reduced to an independent combination of simple forms as in (1). Imoto et al. (1999) introduce a time delay into the transfer, in order to produce periodic type behavior.

The value of stress released during an earthquake can be estimated from the magnitude. Kanamori and Anderson (1975) show that the magnitude $M$ is proportional to the logarithm of the seismic energy $E$ released during an earthquake according to the relation $M=\frac{2}{3} \log _{10} E+$ const. For simplicity, the stress drop during an earthquake is supposed proportional to the square root of the released energy, i.e., $S \propto E^{1 / 2}$. Then, we have the formula

$$
S=10^{0.75\left(M-M_{0}\right)},
$$

where $M_{0}$ is the normalized magnitude. For the subsequent analysis we shall use $M_{0}=5.0$, but the substance of the results are not sensitive to the actual choice.

We shall assume each region to have an exponential risk function, with differing parameters indicating different tectonic properties by region. In other words, the strength (earthquake triggering condition) and tectonic loading rate can differ in each seismic region. Thus we obtain a point process conditional intensity function

$$
\begin{aligned}
\lambda_{i}(t) & =\Psi\left(X_{i}(t)\right) \\
& =\exp \left[\alpha_{i}+v_{i}\left(\rho_{i} t-\sum_{j} \theta_{i j} S^{(j)}(t)\right)\right],
\end{aligned}
$$

for each region $i$, where $\alpha_{i}\left(=\mu_{i}+v_{i} X_{i}(0)\right), v_{i}, \rho_{i}$ and $\theta_{i j}$ are the parameters to be fitted. We choose to parameterize the intensity in this form because it is more amenable to physical intuition. The seemingly excess parameters are in response to fixing $\theta_{i i}=1$ for all $i$. A simpler parameterization (Liu et al., 1998) can be recovered by setting $b_{i}=v_{i} \rho_{i}, c_{i j}=$ $\theta_{i j} / \rho_{i}$. Estimates of the parameters are found by numerically maximizing the log-likelihood

$$
\log L=\sum_{i=1}^{N} \log \lambda\left(t_{i}\right)-\int_{T_{1}}^{T_{2}} \lambda(t) d t,
$$

where the interval $\left(T_{1}, T_{2}\right)$ contains events at times $t_{i}(i=$ $1,2, \ldots, N): T_{1}<t_{1}<t_{2}<\ldots<t_{N}<T_{2}$.

\section{Data and Zoning}

In the earlier analysis by Zheng and Vere-Jones (1994), the Japanese data appear the most complex and most difficult to explain of the three cases (China, Persia and Japan) considered. Generally speaking, as an active island arc structure, Japan seems unlikely to consist of a few relatively welldefined seismic components, but rather of a closely interacting, highly fragmented ensemble within which a few larger units are embedded (Mogi, 1985). The identification of appropriate regions, which have to satisfy both geophysical and statistical requirements, is not in any case trivial, and the regions provided in the following analysis are not claimed to be optimal.

One way that might be used to define regions is by the application of some clustering algorithm, with boundaries drawn equidistant between neighboring clusters. This should recognize implicitly the geophysical structure of the regions, subject to the completeness of the data. An additional consideration is that regions must include sufficient observations to allow the numerical parameter fitting procedure to converge. In this paper we shall attempt to use known tectonic features as the basis of the regions, in order to investigate possible coupling between these features. We should note that, as most events occur along fault lines and other tectonic features, these features must form the interior of such regions as are investigated, rather than performing the perhaps more natural role of region boundaries. Ideally the region boundaries should pass through areas of low seismicity.

The zoning arrived at is shown in Fig. 1. The regions are 1) the western and central parts of the Median Tectonic Line (WMTL), 2) the Chubu/Kinki triangle region (CKT) of central Japan (see, for example, Kanaori et al., 1993), 3) the subduction zone of the Nankai trough (NT), extending north to the "hinge line" (Shimazaki, 1976b), and 4) the Fossa Magna (Oike and Huzita, 1988) and Sagami trough (FMST). Various authors (Kanamori, 1972a; Shimazaki, 1976b; Kanaori et al., 1993, 1994; Pollitz and Sacks, 1997) have argued for the existence of coupling between regions 1, 2 and 3 . This zoning will hopefully enable us to investigate the coupling in a quantitative fashion. Region 4 is included in order to consider its relation to this coupling. Generally, events are assigned to the zone whose defining geophysical feature is nearest to the epicenter. A few of the events in Fig. 1 may seem oddly classified, but the results do not seem to be unduly sensitive to single, small events.

A problem related to zoning is the completeness and reliability of historical records. Shimazaki (1976b) states that the catalog is probably incomplete prior to 1873 , and further it is likely that progressively more earthquakes are missing the further one goes back in time. This must be subsumed in the estimated parameters of the models but the resultant trend will tend to overestimate hazard in simulated forecasts. The best historical records are probably those for the inner and outer seismic belts of central Japan, along with the great offshore events centered on the Nankai and Sagami troughs. We shall make a possibly arbitrary decision to use data from 1585-1997 (although some earlier events going back to 1400 are also used to help determine the initial stress levels) for events with magnitude $M \geq 6.5$. This corresponds to events whose rupture length exceeds the width of 


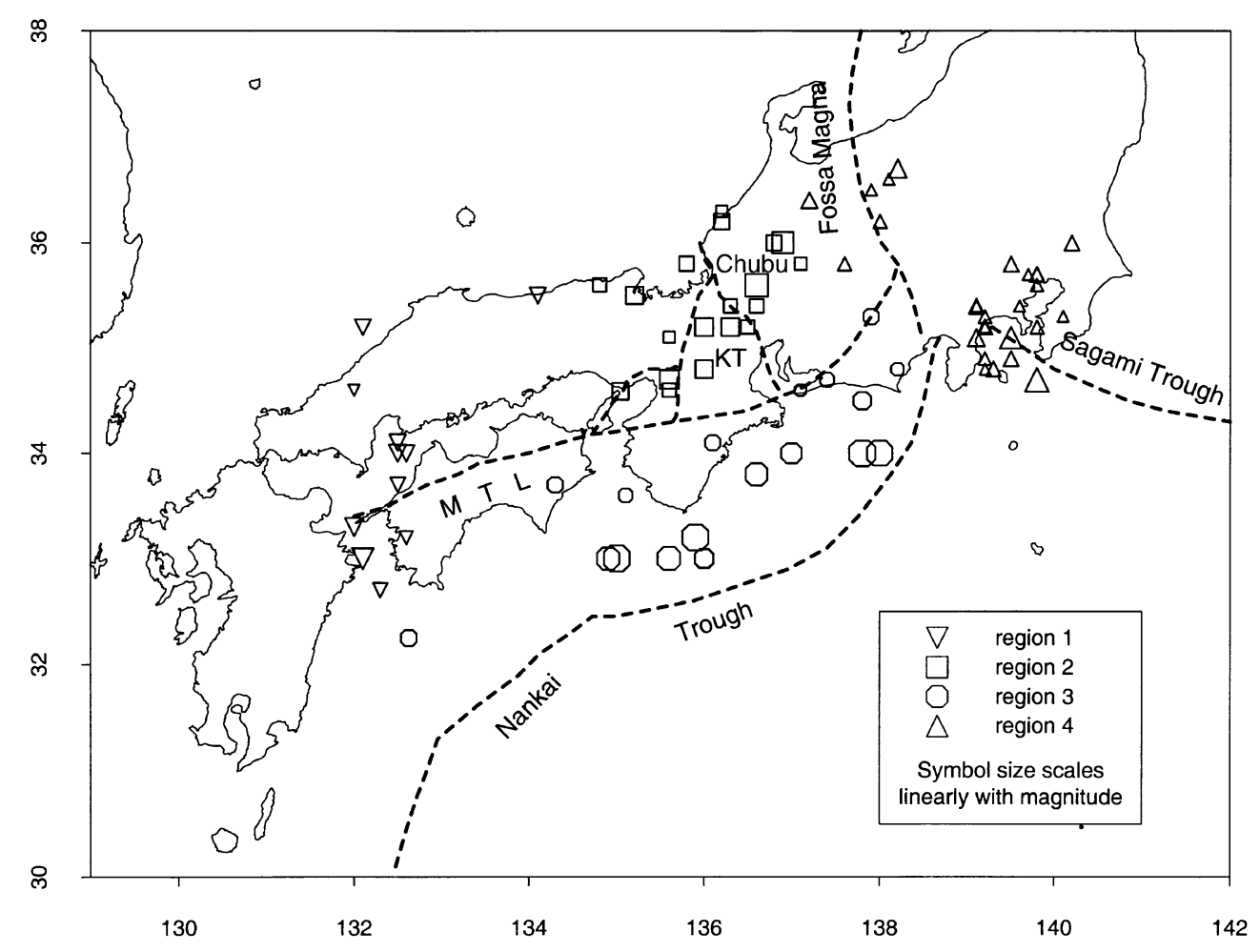

Fig. 1. The epicenter distribution of major earthquakes with magnitude $M \geq 6.5$ (Utsu's catalog). Regions are based on major tectonic features as shown; MTL is the Median Tectonic Line, KT the Kinki triangle, and Chubu refers to the district.

the seismogenic crust (Kanaori, et al., 1993) since, in practice, historical earthquake magnitudes are usually rounded to the nearest 0.5 . The date cutoff enables us to avoid computational complications connected with the absence of events in central Japan 888-1585, which may be a form of seismic quiescence (Kanaori et al., 1992). Thus in (5), $t=0$ corresponds to 1400 , and so $T_{1}=1585-1400=185$, $T_{2}=1997-1400=597$.

Aftershocks were deleted from the catalog using the spacetime window criteria suggested by Gardner and Knopoff (1974), although a satisfactory definition of such a scheme is difficult (Utsu et al., 1995). Fortunately, aftershocks are not present in large numbers due to the magnitude cut-off of $M \geq 6.5$. The resulting data set is provided in Table 1 .

\section{Fitting}

In considering whether parameters differ significantly by region, and depending on the degree of interaction, we have a number of possible models. The choice of the best model in the sense of justified parameters will be based on the Akaike information criterion $(A I C)$, which is defined as

$$
A I C=-2 \log \hat{L}+2 k,
$$

where $\log \hat{L}$ is the maximum log-likelihood for a given model and $k$ is the number of parameters to be fitted in the model (Akaike, 1977). This represents a rough way of compensating for the effect of adding parameters, and is a useful heuristic measure of the relative effectiveness of different models, in avoiding overfitting. For example, the simple stress release model (2) with three parameters as against the Poisson process with only one $(\alpha)$ or the Poisson with exponential trend with two $(\alpha, \beta=\rho v)$ must demonstrate a significantly better fit to justify the additional parameters. Using different combinations of the parameters in (4), we can examine different stress interaction mechanisms. If we suppose, for example, that $\theta_{i j} \neq 0$, we are allowing the events in region $j$ to influence those in region $i$. If we systematically test the various families of interactions, the best model is that with the smallest $A I C$ value. A difference of 2 in $A I C$ is usually considered significant. However, we should note that the $A I C$ values obtained here should be used with some caution, since the amount of historical earthquake data is not very large and the distribution of the log-likelihood is nonstandard.

We must first, before considering the possibility of coupling, determine the best possible model for the regions acting independently. Only if the coupled model, adjusting for its additional parameters through the medium of the $A I C$, is a significantly better fit, can we claim to have a significant degree of coupling. To begin the analysis, the three basic models: Poisson, Poisson with exponential trend, and simple stress release model, are applied to each region. If there is insufficient evidence in the way of $A I C$ values to support the additional parameters of the PWT and SRM, the simpler model will be used for that region. The baseline model will be an independent product of the best region models.

Considering the apparent deficiency of events prior to 1585 , models were fitted by numerically optimising the likelihood over the interval 1585-1997, but known events from 1400 to 1584 were included in the point-process histories. Note that because of the nature of the stress release model, we followed Seno (1979) in treating the Ansei twin events (32 hours apart) as a single event. This accords with our 
Table 1. Historical Japan Earthquake Catalog (by Utsu, 1998). A few earthquakes that are extremely uncertain or perhaps nonexistent are not included in this table.

\begin{tabular}{|c|c|c|c|c|c|c|c|c|c|}
\hline Time & Lat. & Long. & Mag. & Reg. & Time & Lat. & Long. & Mag. & Reg. \\
\hline 1408.01 .12 & 33.00 & 136.00 & 7.5 & 3 & 1854.07 .09 & 34.75 & 136.00 & 7.3 & 2 \\
\hline 1433.10 .29 & 34.90 & 139.50 & 7.0 & 4 & 1854.12 .23 & 34.00 & 137.80 & 8.4 & 3 \\
\hline 1498.09 .11 & 34.00 & 138.00 & 8.3 & 3 & 1854.12 .24 & 33.00 & 135.00 & 8.4 & 3 \\
\hline 1510.09 .11 & 34.60 & 135.60 & 6.8 & 2 & 1854.12 .26 & 33.30 & 132.00 & 7.4 & 1 \\
\hline 1520.03 .25 & 33.00 & 136.00 & 7.4 & 3 & 1855.11 .07 & 34.50 & 137.75 & 7.3 & 3 \\
\hline 1586.01 .18 & 36.00 & 136.90 & 7.8 & 2 & 1855.11 .11 & 35.65 & 139.80 & 6.9 & 4 \\
\hline 1589.03 .21 & 34.80 & 138.20 & 6.7 & 3 & 1857.10 .12 & 34.00 & 132.50 & 7.3 & 1 \\
\hline 1596.09 .05 & 34.65 & 135.60 & 7.5 & 2 & 1858.04 .09 & 36.40 & 137.20 & 7.1 & 4 \\
\hline 1605.02 .03 & 33.00 & 134.90 & 7.9 & 3 & 1872.03 .14 & 35.15 & 132.10 & 7.1 & 1 \\
\hline 1615.06 .26 & 35.70 & 139.70 & 6.5 & 4 & 1891.10 .28 & 35.60 & 136.60 & 8.0 & 2 \\
\hline 1633.03 .01 & 35.20 & 139.20 & 7.0 & 4 & 1894.06 .20 & 35.70 & 139.80 & 7.0 & 4 \\
\hline 1640.11 .23 & 36.30 & 136.20 & 6.5 & 2 & 1894.10 .07 & 35.60 & 139.80 & 6.7 & 4 \\
\hline 1648.06 .13 & 35.20 & 139.20 & 6.5 & 4 & 1899.03 .07 & 34.10 & 136.10 & 7.0 & 3 \\
\hline 1649.03 .17 & 33.70 & 132.50 & 7.0 & 1 & 1899.11 .25 & 32.70 & 132.30 & 6.9 & 1 \\
\hline 1649.07 .30 & 35.80 & 139.50 & 7.0 & 4 & 1905.06 .02 & 34.10 & 132.50 & 7.2 & 1 \\
\hline 1662.06 .16 & 35.20 & 135.95 & 7.4 & 2 & 1906.01 .21 & 34.00 & 137.00 & 7.6 & 3 \\
\hline $1685.04 .-^{*}$ & 34.60 & 137.10 & 6.5 & 3 & 1909.08 .14 & 35.40 & 136.30 & 6.8 & 2 \\
\hline 1686.01 .04 & 34.00 & 132.60 & 7.2 & 1 & 1918.11 .11 & 36.50 & 137.90 & 6.5 & 4 \\
\hline 1686.10 .03 & 34.70 & 137.40 & 6.8 & 3 & 1921.12 .08 & 36.00 & 140.20 & 7.0 & 4 \\
\hline 1697.11 .25 & 35.40 & 139.60 & 6.5 & 4 & 1922.04 .26 & 35.20 & 139.80 & 6.8 & 4 \\
\hline 1703.12 .31 & 34.70 & 139.80 & 8.1 & 4 & 1923.09 .01 & 35.10 & 139.50 & 7.9 & 4 \\
\hline 1707.10 .28 & 33.20 & 135.90 & 8.4 & 3 & 1925.05 .23 & 35.60 & 134.80 & 6.8 & 2 \\
\hline 1715.02 .02 & 35.40 & 136.60 & 6.8 & 2 & 1927.03 .07 & 35.53 & 135.15 & 7.3 & 2 \\
\hline 1718.08 .22 & 35.30 & 137.90 & 7.0 & 3 & 1930.11 .26 & 35.08 & 139.05 & 7.3 & 4 \\
\hline 1749.05 .25 & 33.20 & 132.60 & 6.8 & 1 & 1931.11 .02 & 32.25 & 132.63 & 7.1 & 3 \\
\hline 1769.08 .29 & 33.00 & 132.10 & 7.8 & 1 & 1938.01 .12 & 33.58 & 135.07 & 6.8 & 3 \\
\hline 1778.02 .14 & 34.60 & 132.00 & 6.5 & 1 & 1943.09 .10 & 35.52 & 134.08 & 7.2 & 2 \\
\hline 1782.08 .23 & 35.40 & 139.10 & 7.0 & 4 & 1944.12 .07 & 33.80 & 136.62 & 7.9 & 3 \\
\hline 1789.05 .11 & 33.70 & 134.30 & 7.0 & 3 & 1946.12 .21 & 33.03 & 135.62 & 8.0 & 3 \\
\hline 1791.07 .23 & 36.20 & 138.00 & 6.8 & 4 & 1948.06 .28 & 36.17 & 136.20 & 7.1 & 2 \\
\hline 1801.05 .27 & 35.30 & 140.10 & 6.5 & 4 & 1961.08 .19 & 36.02 & 136.77 & 7.0 & 2 \\
\hline 1802.11 .18 & 35.20 & 136.50 & 6.8 & 2 & 1963.03 .27 & 35.78 & 135.77 & 6.9 & 2 \\
\hline 1819.08 .02 & 35.20 & 136.30 & 7.3 & 2 & 1969.09 .09 & 35.78 & 137.07 & 6.6 & 2 \\
\hline 1830.08 .19 & 35.10 & 135.60 & 6.5 & 2 & 1978.01 .14 & 34.77 & 139.25 & 7.0 & 4 \\
\hline 1843.03 .09 & 35.35 & 139.10 & 6.5 & 4 & 1980.06 .29 & 34.92 & 139.23 & 6.7 & 4 \\
\hline 1847.05 .08 & 36.70 & 138.20 & 7.4 & 4 & 1984.09 .14 & 35.82 & 137.56 & 6.8 & 4 \\
\hline 1853.01 .26 & 36.60 & 138.10 & 6.5 & 4 & 1990.02 .20 & 34.76 & 139.23 & 6.5 & 4 \\
\hline 1853.03 .11 & 35.30 & 139.15 & 6.7 & 4 & 1995.01.17 & 34.59 & 135.04 & 7.2 & 2 \\
\hline
\end{tabular}

*The 15 th was used as the day for the 1685 event in calculations. 
Table 2. Baseline model for independent regions. The fitted parameters are those of the simple stress release model for each region.

\begin{tabular}{lcccccc}
\hline \multicolumn{1}{c}{ Region $i$} & $\alpha_{i}$ & $\nu_{i}$ & $\rho_{i}$ & $A I C_{\mathrm{PP}}$ & $A I C_{\mathrm{PWT}}$ & $A I C_{\mathrm{SRM}}$ \\
\hline 1 (WMTL) & -10.306 & 0.0147 & 1.6945 & 104.03 & 105.51 & 103.46 \\
2 (CKT) & -4.814 & 0.0013 & 4.1488 & 151.22 & 150.76 & 152.55 \\
3 (NT) & -4.972 & 0.0033 & 4.0931 & 131.82 & 133.59 & 132.89 \\
4 (FMST) & -8.939 & 0.0084 & 2.8915 & 198.42 & 196.15 & 189.49 \\
\hline
\end{tabular}

object of forecasting risk over a period of decades.

With regions as given in Fig. 1, the results are set out in Table 2. It is easy to see that the stress release model is better (has smaller $A I C$ ) than the Poisson process in regions 1 and 4, and better than the Poisson with trend except in region 2 . Thus, if we treat the regions separately, fitting regions 1 and 4 by the stress release model, region 2 by the Poisson with trend, and region 3 by the Poisson process, we obtain our baseline model, on which we shall seek to improve. This baseline model has $A I C=575.53$. Note that an aggregation of four independent simple stress release models, which we shall use below, has a combined $A I C=$ 578.39, with 6 additional parameters.

The first question is whether there is any justification to allow $\rho_{i}$, the tectonic input rate, to differ by region. To test this, a model of four independent stress release processes, with $\rho_{i}=\rho$, for all $i$ (one free parameter for tectonic input) was fitted. This produced an AIC of 577.59, less than the AIC of 578.39 resulting from the extra free parameters. Hence we conclude that, in the stress release setup, there is insufficient evidence to support variations in $\rho_{i}$. All subsequent fitted models will have $\rho_{i}=\rho$, a common value. Due to the complicated makeup of the area, with each region being an ensemble of many closely interacting fragments, it is obvious that loading would be a complex aggregation of many elements. Also, loading imbalances could be equalized on a scale below that of the large events considered in the study, or through aseismic slip (Mogi, 1985). Thus a constant loading rate could best explain the portion of the tectonic loading that acts to produce large earthquakes.

We can now consider the possible inter-regional interactions. We have already considered the case of independent stress release processes (the zero-range model). If we allow all possible interactions, then we have a long range model, while if we do not allow direct interactions between regions 1 and 4, we obtain a short range model. As we see in Table 3, neither of these improves on the results from the baseline model (or indeed the zero-range model). Since region 4 is not usually considered in studies of this area, we next treat it as an independent stress release process, and allow all interactions among the remaining regions. As we can see from the resultant increase in the $A I C$, it appears that there is a significant degree of interaction between region 4 and the remaining regions. However, when region 1 was subjected to the same test, we obtained a very significant decrease in $A I C$, leading us to conclude that there is insufficient evidence linking events in region 1 with events in the remaining regions. Removing these interactions leaves us with a transfer
Table 3. Inter-regional interaction models.

\begin{tabular}{lrc}
\hline Interactions & \multicolumn{1}{c}{$k$} & \multicolumn{1}{c}{$A I C$} \\
\hline Baseline & 9 & 575.53 \\
Zero range & 9 & 577.59 \\
Long range & 21 & 578.83 \\
Short range & 19 & 577.28 \\
Region 4 independent & 15 & 580.44 \\
Region 1 independent & 15 & 569.86 \\
\hline
\end{tabular}

matrix of the form

$$
\Theta=\left(\theta_{i j}\right)=\left(\begin{array}{cccc}
1 & 0 & 0 & 0 \\
0 & 1 & \theta_{23} & \theta_{24} \\
0 & \theta_{32} & 1 & \theta_{34} \\
0 & \theta_{42} & \theta_{43} & 1
\end{array}\right) .
$$

With at most 6 free parameters, there are $2^{6}=64$ possible models. Fitting all of these, we obtained our best model, as shown in Table 4, with an AIC $=565.78$ with 12 parameters. Although this may seem a large number of parameters, it should be recalled that additional parameters must justify themselves statistically. As an analogy, one could think of a spring-block model, with springs linking every pair of blocks, and with springs connected between each block and a driving plate (see, for example, Rundle et al., 1995). If each block has a random initial displacement, and the spring constants are allowed to vary, the number of parameters is comparable. Here we have only four types of parameter, but we estimate each value separately, provided that the differences are shown to be significant. The model indicates that events in region 4 transfer stress to both regions 2 and 3, while events in region 2 transfer stress to region 4 . Thus, events in region 2 may encourage events in region 3 , via the intermediacy of events in region 4.

Of the remaining 63 models, the $A I C$ values divide them into two groups. The first group have $A I C$ within 5 or so of the best model (often but little inferior), while the $A I C$ values of the second group are greater than those of all of the first group. The first group is distinguished by having interactions that allow the transfer of stress between region 2 and region 4 , in accordance with the observations of Oike and Huzita (1988), and from region 2 to region 3 (it can be a two-step transfer via region 4$)$. In other words $\theta_{42} \neq 0, \theta_{24} \neq 0$ and at least one of $\theta_{32}$ and $\theta_{34}$ is nonzero. 
Table 4. The estimated values of the parameters of the best linked model.

\begin{tabular}{lccccccc}
\hline \multicolumn{1}{c}{ Region $i$} & $\alpha_{i}$ & $v_{i}$ & $\rho_{i}$ & $\theta_{i 1}$ & $\theta_{i 2}$ & $\theta_{i 3}$ & $\theta_{i 4}$ \\
\hline 1 (WMTL) & -10.246 & 0.0148 & 1.676 & 1 & 0 & 0 & 0 \\
2 (CKT) & -4.539 & 0.0025 & 1.676 & 0 & 1 & 0 & -0.6579 \\
3 (NT) & -3.163 & 0.0076 & 1.676 & 0 & 0 & 1 & -0.9639 \\
4 (FMST) & -9.460 & 0.0133 & 1.676 & 0 & -0.5839 & 0 & 1 \\
\hline
\end{tabular}
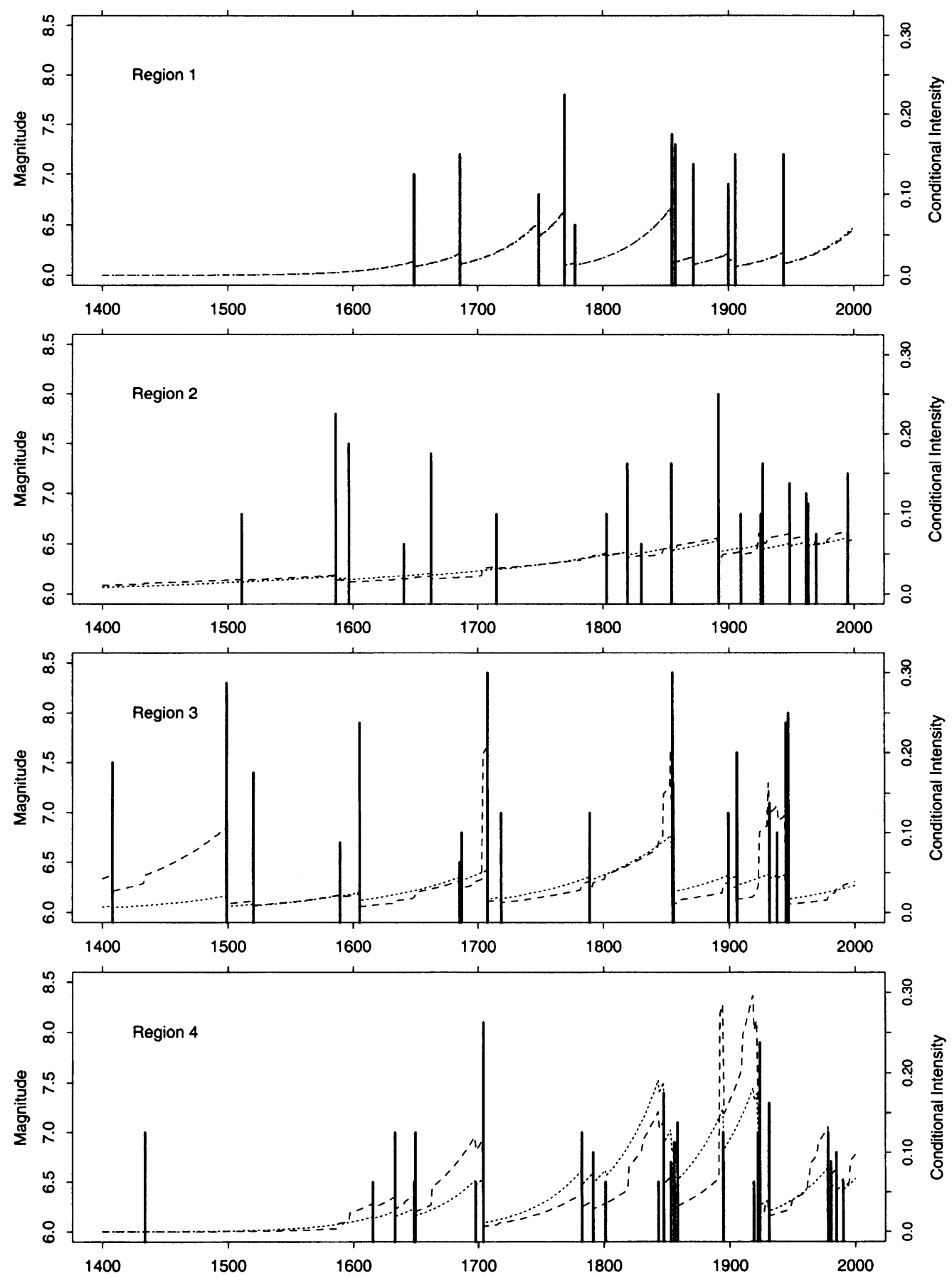

Fig. 2. Regional intensities from the coupled model (dashed line) and the independent model (dotted line). The vertical lines are the magnitudes of the historical events. 

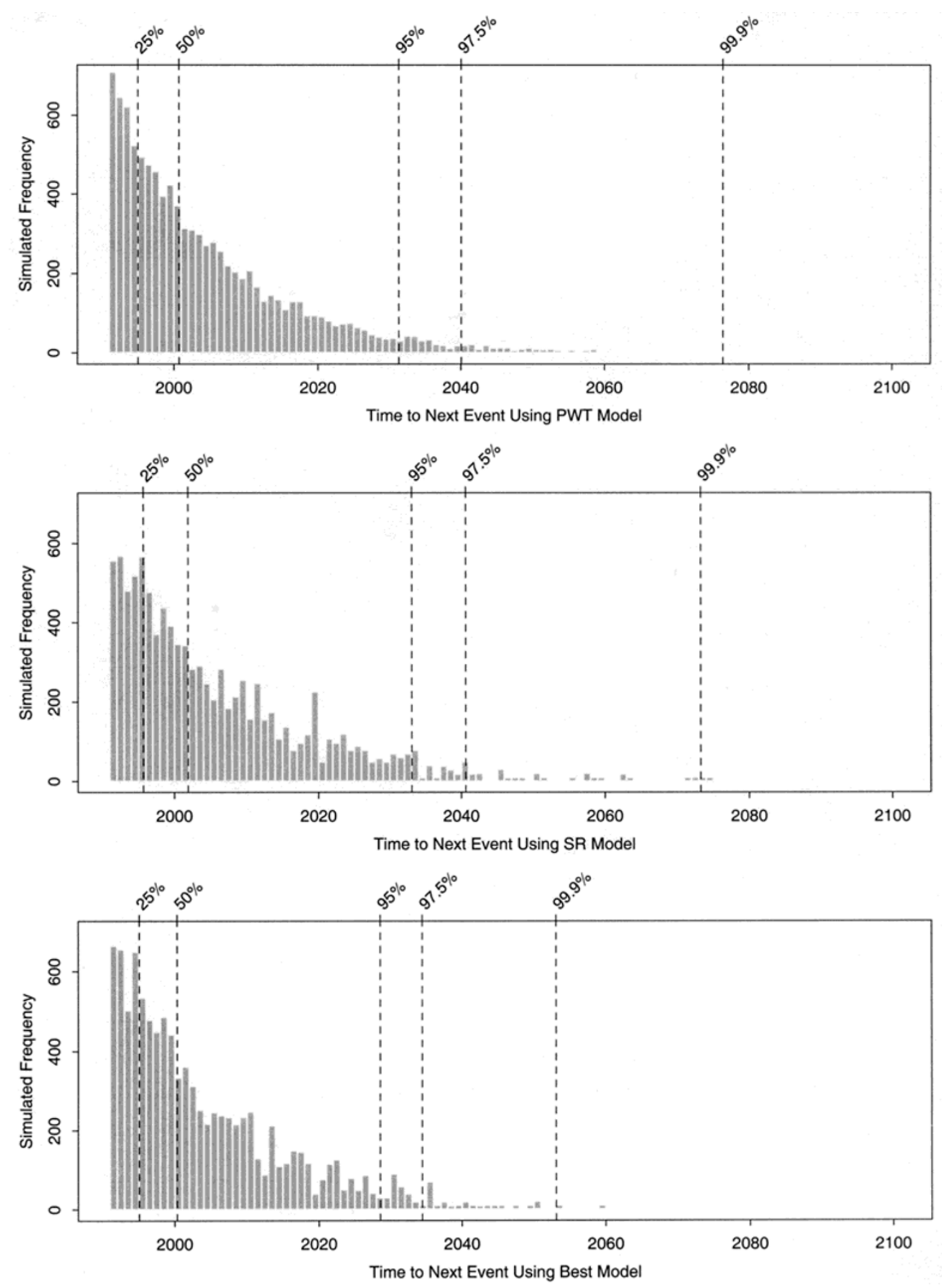

Fig. 3. Simulated time of next event in region 2 from 1991 (10000 simulations). The top two graphs show the time of the next event in region 2 using the PWT model and SRM model of Table 2, respectively, while the bottom graph is the time of the next event in region 2 from the model in Table 4 . The vertical dashed lines show various quantiles of the simulated times.

\section{Results and Discussion}

Since the model in Table 4 with $A I C=565.78$ is far better than the baseline model with $A I C=575.53$, there is evidence to suggest long-range interaction of elastic stress between different regions. Since the off-diagonal elements of $\Theta$ are negative, this indicates that events, while reducing the stress in their own region, increase the stress elsewhere. Note that stress is not conserved. This transferred stress increases the hazard in the receiving region, and hence earthquakes may trigger, in a stochastic sense, further earthquakes in quite distant regions. In particular, there seems to be evidence that events in region 3 are partly due to events in region 4 (and those in region 2). The events in region 1, on the other hand, seem neither to trigger, nor to be triggered by, events elsewhere.

The calculated conditional intensities using the fitted parameters from the best linked stress release model are shown in Fig. 2. They clearly show how the interactions between the regions increase the likelihood of events being triggered.

To further demonstrate the effect, and test the validity of the model, we can take the catalog from 1400 to 1991, and repeatedly simulate (Wang et al., 1991) the best model for- 


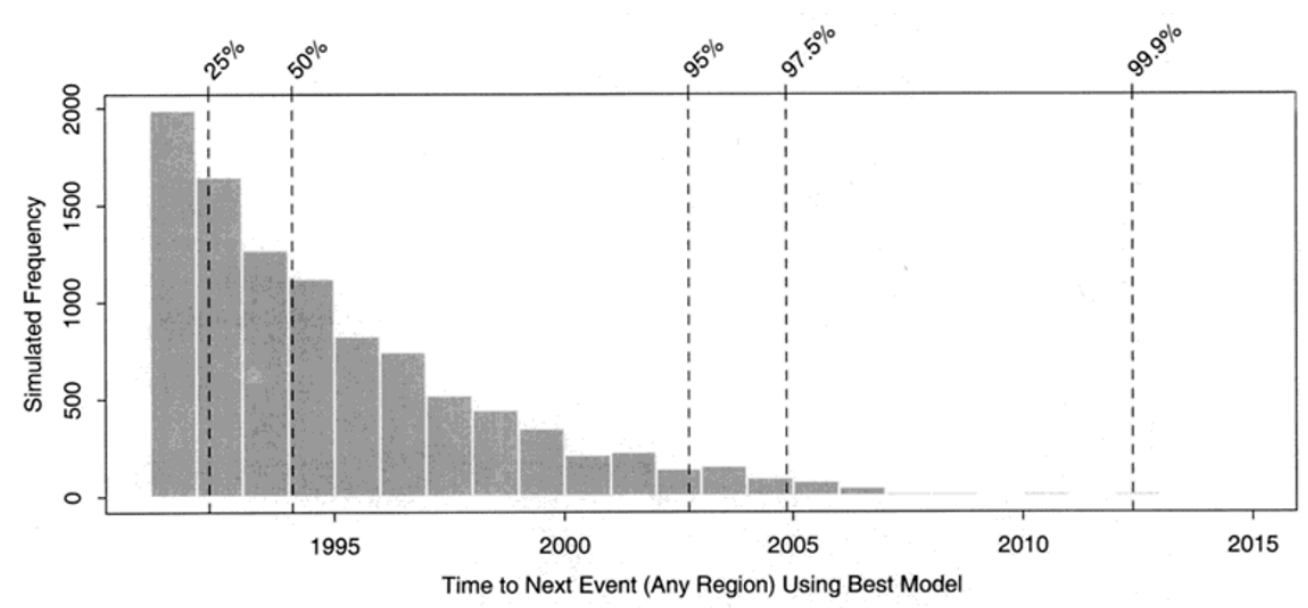

Fig. 4. Simulated time of next event from 1991 (10000 simulations) in any region. The vertical dashed lines show various quantiles of the simulated times.

ward to forecast the time of the next large event, and the time of the next large event in region 2 . This can be compared with models for region 2 independently. Over long periods, an information gain can be calculated (Vere-Jones, 1998), but here because of the paucity of data we shall restrict ourselves to considering only the next event. The actual next event was the Kobe earthquake (17 Jan, 1995) in region 2. The parameters of the simulated models will, of course, differ slightly from those in Tables 2 and 4 as they will not be fitted to the Kobe event. The results are shown in Fig. 3. We see that the best linked model enhances the likelihood, as measured by the tail probabilities shown, of an earlier event in region 2. This is because it is able to use the large number of recent events in region 4 directly through the parameter $\theta_{24}(<0$, indicating an excitation effect). The same effect can be seen in Fig. 2. Although the result is not a strong one, it does indicate that the model is proceeding in the right direction. In order to further enhance forecasting, it needs to take into account aftershocks, thus increasing the amount of useable data. Figure 4 shows the corresponding forecast for all regions using the model in Table 4.

Ando (1975) identifies a Phillipines sea loading cycle of 180 years. In a point process model with intensity $\lambda(t)$, the mean number of events in the period $[0, t]$ is $\mu(t)=$ $\int_{0}^{t} \lambda(s) d t$. If we restart after an event, the length of the reloading cycle can be identified as $t$, such that $\mu(t)=1$, in which only the tectonic loading part of the intensity is considered. We find that

$$
\mu(t)=\frac{e_{i}^{\alpha}}{v_{i} \rho_{i}}\left(e^{v_{i} \rho_{i} t}-1\right)
$$

which yields $t=80$ years using the parameters from Table 2 . The discrepancy is explained by including smaller events in our study.

Pollitz and Sacks (1997) claim a typical time scale for significant post seismic effects in Japan to be 30-50 years, and that post-seismic effects are dominant over reloading effects for the first $1 / 4-1 / 3$ of the reloading cycle. We find from (3) and Table 4 that a magnitude 7.5 event in region 4 will transfer an amount of stress $\theta_{34} \times 10^{0.75(7.5-5.0)}$ to region 3. This is equivalent to 72 years of tectonic input, or 0.4 of the reloading cycle, whereas a magnitude 7.0 event is equivalent to 18 years of tectonic input.

Shimazaki (1976b) calculated that the stress reloaded by intraplate events increases at a slow rate. The different values of the common $\rho$ for the linked model, and that of the zerorange model (2.975) indicate that some $44 \%$ of the initiating stress is derived from transfer, the remainder from tectonic input.

Kanaori et al. (1993) note that events in the Chubu/Kinki triangle region are followed coevaly or slightly afterward by events on the MTL, and then some 30 years or less later by an event in the Nankai trough. The first assertion appears to be a statistical artifact, and the second is hard to test, since we have 16 events in region 3, or an average of one every 26 years. However, when contrasted with the claim of Thatcher (1984) that the events along the MTL are triggered by Nankai trough events, our results, in particular the direction of stress transfer, certainly support the views in Kanaori et al. (1993). Kanaori et al. (1993) also claim that the MTL is subject to aseismic slip, which the independence result for region 1 does not contradict.

\section{Conclusions}

We have proposed a linked stress release model, incorporating stress transfer and spatial interaction. This model fits the Japanese data better than a collection of independent simple models. The results provide a possible hint that the crust may lie in a near-critical state due to a long-range interaction of elastic stress, and provide a paradigm whereby spatio-temporal complexity of seismicity is related to both the dynamics and heterogeneities in a region. An earthquake could trigger (within a period of a few years) another in a quite distant region. The fitted parameters provide statistical evidence, with some quantification, for proposed couplings between tectonic regions. Using the fitted parameters and following the general lines for the simulation of point processes presented by Ogata (1981), we can forecast the longterm risk in a region: A post-1991 example resulted in an enhanced hazard level in region 2, corresponding to the 1995 Kobe event. 
Acknowledgments. M. Imoto and an anonymous referee made several suggestions, particularly concerning zoning, that much improved the structure of the paper. We are also grateful to X. Zheng, H. Takayasu, Y. Shi and especially D. Vere-Jones for many valuable discussions, and to T. Utsu and Y. Ogata for kindly supplying us the latest Japanese earthquake catalog. This work was supported by the Marsden Fund administered by the Royal Society of New Zealand. M. Bebbington was supported by a Raybould Visiting Fellowship at The University of Queensland.

\section{References}

Akaike, H., On entropy maximization principle, in Applications of Statistics, edited by P. R. Krishnaiah, pp. 27-41, North Holland, Amsterdam, 1977.

Ando, M., Source mechanism and tectonic significance of historical earthquakes along the Nankai trough, Japan, Tectonophysics, 27, 119-140, 1975.

Bak, P. and C. Tang, Earthquakes as a self-organized critical phenomenon, J. Geophys. Res., 94, 15635-15637, 1989.

Ben-Zion, Y. and J. R. Rice, Dynamic simulations of slip on a smooth fault in an elastic solid, J. Geophys. Res., 102, 17771-17784, 1997.

Bufe, C. G. and D. J. Varnes, Predictive modelling of the seismic cycle of the greater San Francisco Bay region, J. Geophys. Res., 98, 9871-9883, 1993.

Chen, K., P. Bak, and S. P. Obukhov, Self-organized criticality in a crackpropagation model of earthquakes, Phys. Rev. A, 43, 625-630, 1991.

Daley, D. J. and D. Vere-Jones, An Introduction to the Theory of Point Processes, 702 pp., Springer, Berlin, 1988.

Gabrielov, A. and W. I. Newman, Seismicity modeling and earthquake prediction: A review, in Nonlinear Dynamics and Predictability of Geophysical Phenomena, edited by W. I. Newman, A. Gabrielov, and D. L. Turcotte, pp. 7-13, Am. Geophy. Union, Washington, D.C., 1994.

Gardner, J. K. and L. Knopoff, Is the sequence of earthquakes in southern California, with aftershocks removed, Poissonian?, Bull. Seismol. Soc. Am., 64, 1363-1367, 1974.

Gutenberg, B. and C. F. Richter, Seismicity of the Earth and Associated Phenomenon, 2nd edition, 310 pp., Princeton University Press, Princeton, 1954.

Hill, D. P., P. A. Reasenberg, A. Michael, W. J. Arabaz, G. Beroza, D. Brumbaugh, J. N. Brune, R. Castro, S. Davis, D. dePolo, W. L. Ellsworth, J. Gomberg, S. Harmsen, L. House, S. M. Jackson, M. J. S. Johnston, L. Jones, R. Keller, S. Malone, L. Munguia, S. Nava, J. C. Pechmann, A. Sanford, R. W. Simpson, R. B. Smith, M. Stark, M. Stickney, A. Vidal, S. Walter, V. Wong, and J. Zollweg, Seismicity remotely triggered by the magnitude 7.3 Landers, California, earthquake, Science, 260, 1617-1623, 1993.

Imoto, M., K. Maeda, and A. Yoshida, Use of statistical models to analyze periodic seismicity observed for clusters in the Kanto region, central Japan, PAGEOPH, 155, 609-624, 1999.

Ito, K. and M. Matsuzaki, Earthquakes as self-organized critical phenomena, J. Geophys. Res., 95, 6853-6860, 1990.

Kagan, Y. Y., Observational evidence for earthquakes as a non-linear dynamic process, Physica D, 77, 160-192, 1994.

Kanamori, H., Tectonic implications of the 1944 Tonankai and the 1946 Nankaido earthquakes, Phys. Earth Planet. Inter, 5, 129-139, 1972a.

Kanamori, H., Relations among tectonic stress, great earthquakes and earthquake swarm, Tectonophysics, 14, 1-12, $1972 \mathrm{~b}$.

Kanamori, H. and D. L. Anderson, Theoretical basis of some empirical relations in seismology, Bull. Seismol. Soc. Am., 65, 1073-1095, 1975.

Kanaori, Y., S. Kawakami, and K. Yairi, Space-time distribution patterns of destructive earthquakes in the inner belt of central Japan: Activity intervals and locations of earthquakes, Eng. Geol., 31, 209-230, 1991.

Kanaori, Y., S. Kawakami, and K. Yairi, Space-time distribution patterns of destructive earthquakes in the inner belt of central Japan (part 2): Moment-release rates and earthquake prediction, Eng. Geol., 32, 113122, 1992.

Kanaori, Y., S. Kawakami, and K. Yairi, Space-time correlations between inland earthquakes in central Japan and great offshore earthquakes along the Nankai trough: Implication for destructive earthquake prediction, Eng. Geol., 33, 289-303, 1993.

Kanaori, Y., S. Kawakami, and K. Yairi. Seismotectonics of the Median Tectonic Line in southwest Japan: Implications for coupling among major fault systems, PAGEOPH, 142, 589-607, 1994.
Knopoff, L., A stochastic model for the occurrence of main-sequence earthquakes, Rev. Geophys. Space Phys., 9, 175-188, 1971.

Liu, J., D. Vere-Jones, L. Ma, Y. Shi, and J. Zhuang, The principle of coupled stress release model and its applications, Acta Seismologica Sinica, 11 273-281, 1998

Lu, C., H. Takayasu, A. Tretyakov, M. Takayasu, and S. Yumoto, Selforganized criticality in a block lattice model of the brittle crust, Phys. Lett. A, 242, 349-354, 1998.

Main, I., Statistical physics, seismogenesis, and seismic hazard, Rev. Geophys., 34, 433-462, 1996.

Mogi, K., Earthquake Prediction, 355 pp., Academic Press, Tokyo, 1985.

Ogata, Y., On Lewis's simulation method for point processes, IEEE Trans. Inf. Theory, IT-27, 23-31, 1981.

Oike, K. and K. Huzita, Relation between characteristics of seismic activity and neotectonics in Honshu, Japan, Tectonophysics, 148, 115-130, 1988

Pollitz, F. F. and I. S. Sacks, The 1995 Kobe, Japan, earthquake: A longdelayed aftershock of the offshore 1944 Tonankai and 1946 Nankaido earthquakes, Bull. Seismol. Soc. Amer., 87, 1-10, 1997.

Reid, H. F., The mechanism of the earthquake, in The California Earthquake of April 18, 1906, Report of the State Earthquake Investigation Commission, Vol. 2, pp. 16-28, Carnegie Institute of Washington, Washington, D.C., 1910.

Rundle, J. B., W. Klein, S. Gross, and D. L. Turcotte, Boltzman fluctuations in numerical simulations of nonequilibrium lattice threshold systems, Phys. Rev. Lett., 75, 1658-1661, 1995.

Seno, T., Pattern of intraplate seismicity in Southwest Japan before and after great interplate earthquakes, Tectonophysics, 57, 267-283, 1979.

Shi, Y., J. Liu, D. Vere-Jones, J. Zhuang, and L. Ma, Application of mechanical and statistical models to the study of the seismicity of synthetic earthquakes and the prediction of natural ones, Acta Seismologica Sinica, 11, 421-430, 1998.

Shimazaki, K., Intra-plate seismicity gap along the Median Tectonic Line and oblique plate convergence in southwest Japan, Tectonophysics, $\mathbf{3 1}$ 139-156, 1976a.

Shimazaki, K., Intra-plate seismicity and inter-plate earthquakes: Historical activity in southwest Japan, Tectonophysics, 33, 33-42, 1976b.

Shimazaki, K. and T. Nakata, Time-predictable recurrence model for large earthquakes, Geophys. Res. Lett., 7, 279-282, 1980.

Sornette, D. and C. G. Sammis, Complex critical exponents from renormalization group theory of earthquakes, J. Phys. I. France, 5, 607-619, 1995.

Takayasu, H., Fractals in the Physical Sciences, 170 pp., Manchester University Press, Manchester, 1990.

Thatcher, W., The earthquake deformation cycle at the Nankai trough, $J$. Geophys. Res., 89, 3087-3101, 1984.

Utsu, T., Historical Earthquakes Accompanied by Damage in Japan, Communication by Y. Ogata, 1998.

Utsu, T., Y. Ogata, and R. S. Matsu' ura, The centenary of the Omori formula for a decay law of aftershock activity, J. Phys. Earth, 43, 1-33, 1995.

Vere-Jones, D., A branching model for crack propagation, $P A G E O P H, \mathbf{1 1 4}$, 711-726, 1976.

Vere-Jones, D., Earthquake prediction: A statistician's view, J. Phys. Earth, 26, 129-146, 1978 .

Vere-Jones, D., Probabilities and information gain for earthquake forecasting, Computational Seismology, 30, 249-263, 1998.

Vere-Jones, D. and Y. L. Deng, A point process analysis of historical earthquakes from North China, Earthq. Res. China, 2, 165-181, 1988.

Wang, A. L., D. Vere-Jones, and X. Zheng, Simulation and estimation procedures for stress release models, in Stochastic Processes and Their Applications, edited by M. J. Beckman, M. N. Gopalan, and R. Subramani, Lecture notes in economics and mathematical systems 370, pp. 11-27, Springer-Verlag, Berlin, 1991.

Zhao, Z., K. Oike, K. Matsumura, and Y. Ishikawa, Stress field in the continental part of China derived from temporal variations of seismic activity, Tectonophysics, 178, 357-372, 1990.

Zheng, X. and D. Vere-Jones, Application of stress release models to historical earthquakes from North China, PAGEOPH, 135, 559-576, 1991.

Zheng, X. and D. Vere-Jones, Further applications of the stochastic stress release model to historical earthquake data, Tectonophysics, 229, 101$121,1994$.

C. Lu (e-mail: cslu@mcs.vuw.ac.nz), D. Harte, and M. Bebbington 